\title{
TIROIDES
}

\section{Presentación oral}

\section{Cambios en el perfil tiroideo de la gestante obesa}

\section{Seudónimo: Linis}

Objetivo: evaluar los niveles de hormonas tiroideas en una población de mujeres gestantes con obesidad con un embarazo a término y con peso normal o normopeso, que asistieron a una institución de nivel III de atención.

Materiales y métodos: un estudio de casos y controles realizado en una entidad de salud nivel III de complejidad, en el suroccidente colombiano. Se seleccionaron 40 mujeres entre 18-37 años, con embarazo único a término, sin diagnóstico previo de comorbilidades. Las gestantes se clasificaron en dos grupos: a) 20 gestantes con peso pregestacional normal y b) 20 gestantes obesas pregestacionales. En el suero materno, se determinaron los niveles de la hormona estimulante de la tiroides (TSH), T4 libre, T3 total y T3 libre. La comparación entre los grupos se realizó por medio de la prueba $t$-Student. Los valores se expresaron como la media \pm el error estándar.
Resultados: los niveles de TSH no presentaron diferencias entre los grupos. Sin embargo, el grupo de mujeres con obesidad pregestacional mostró una disminución significativa de los niveles de T4 libre en comparación con las gestantes normopeso $(0,896 \pm 0,041 \mathrm{ng} / \mathrm{dL}$ frente a 1,043 $\pm 0,044 \mathrm{ng} /$ $\mathrm{dL} ; p<0,03)$. La T3 total presentó niveles más elevados en el grupo con obesidad pregestacional en comparación al grupo con normopeso $(1,908 \pm 0,066 \mathrm{ng} / \mathrm{mL}$ frente a 1,625 $\pm 0,049$ $\mathrm{ng} / \mathrm{mL} ; p<0,003)$. En contraste, la T3 libre no mostró diferencias entre los grupos.

Conclusión: la obesidad pregestacional afecta los niveles de T4 libre y T3 total, lo que sugiere que esta patología altera de forma directa el eje regulatorio de las hormonas tiroideas.

Palabras claves: función tiroidea, obesidad, embarazo.

\section{Presentación oral}

\section{Characteristics of Patients with Thyroid Cancer Evaluated by positron Emission Tomography in Imbanaco Medical Center}

\section{Pseudonym: PET}

Introduction: Positron-emission tomography (PET/CT) is a useful tool for the assessment of tumoral extension and behavior in several types of cancers. Its main indication in patients with differentiated thyroid cancer (DTC) consists in those previously treated with total thyroidectomy and radioiodine, who show elevation of serum thyroglobulin (Tg) levels and negative findings in radioiodine scan (RS) or ultra-sound, and those with aggressive variants (such as anaplastic or poorly differentiated entities).

Methods: We conducted a retrospective analysis of 62 patients ( 44 of whom were female) with a mean of 51 years of age; papillary carcinoma was found in $80.2 \%$ of them, while follicular, anaplastic, and Hurtle cell variants were present in 9.1\%, $1.5 \%$, and $1.5 \%$ of them, respectively. The mean time between diagnosis of DTC and PET/CT was 7.2 years. PET/CT scans were retrieved between 2016 and 2018, with none of them performed under TSH stimulation. Thirty-eight patients were treated with radioiodine, nine (23\%) of whom received a cumulative dose $>600$ millicuries (mCi). Mean Tg concentration was $209 \mathrm{ng} / \mathrm{mL}$.

Results: Twenty-two patients showed Tg concentrations $>10 \mathrm{ng} / \mathrm{mL}$, of whom 14 (64\%) had positive findings in PET/ CT. Six patients had $\mathrm{Tg}<10 \mathrm{ng} / \mathrm{mL}$ and PET/CT positive. PET/ CT yielded positive findings in 38 patients in total, with findings of uptake in thyroid bed (TB) in seven of them, along with 19 cases of compromise of cervical lymph nodes (CLN) and 14 cases of metastases (12 in the lung, 1 in the mediastinum, and 1 in bone). Five patients had more than one lesion. Mean standardized uptake value (SUVmax) was 15.9+/-25.

Conclusion: In our series, PET/CT was positive in twothirds of patients with elevated $\mathrm{Tg}$ and suspicion of recurrence. Nevertheless, half of those PET/CT-positive patients showed Tg concentrations $<10 \mathrm{ng} / \mathrm{mL}$. The TB and CLN were the most frequent sites of recurrence (68\%) and metastases in the $37 \%$ of scans. 


\title{
Prevalencia del hipotiroidismo y caracterización clínica en gestantes del Eje Cafetero (Colombia), 2014-2017: estudio transversal
}

\author{
Seudónimo: Negroncho 1
}

Objetivo: conocer la prevalencia del hipotiroidismo en una población de mujeres gestantes del Eje Cafetero (Colombia).

Metodología: se realizó un estudio de corte transversal descriptivo. Se incluyeron mujeres embarazadas de primer ingreso al control prenatal. A todas las gestantes se les realizó la prueba de perfil tiroideo con base en las recomendaciones de la Asociación Americana de Tiroides (ATA, por sus siglas en inglés). Se incluyeron gestantes mayores de edad, con embarazo único, historia clínica electrónica y sin enfermedad tiroidea preexistente que aceptaron participar en el estudio. Se excluyeron las gestantes con enfermedad neoplásica o hematológica, insuficiencia renal crónica o con anormalidades fetales congénitas. El estudio se realizó en tres clínicas del Eje Cafetero nivel III, entre el 2014 y 2017. Se realizó un muestreo aleatorio sistemático consecutivo. Se midieron variables sociodemográficas, la sintomatología de hipotiroidismo y los antecedentes de salud sexual y reproductiva. Se realizó un análisis descriptivo de la información utilizando medidas de frecuencia absoluta y relativa.

Resultados: de un total de 579 mujeres seleccionadas a participar, se analizaron finalmente 467 (80,65\%). La edad promedio fue de 24,85 años (desviación estándar [DE] $\pm 5,3$ ). La prevalencia del hipotiroidismo en el grupo estudiado fue del 38,75 \% ( $\mathrm{n}=181 / 479), 22,69 \%(\mathrm{n}=106)$ con hipotiroidismo clínico y $15,99 \%(n=75)$ con hipotiroidismo subclínico, de las cuales el $12,41 \%(n=58)$ presentaron un trastorno tiroideo autoinmune.

Conclusión: las gestantes del Eje Cafetero presentan una alta prevalencia de hipotiroidismo, mayor a lo reportado en otras zonas del mundo. Se hace necesario promocionar intervenciones oportunas para establecer un pronto diagnóstico y una terapéutica apropiada.

Palabras clave: hipotiroidismo, embarazo, factores de riesgo, diagnóstico, prevalencia, tiroides.

Presentación oral

\section{Proyecto genoma del cáncer en Colombia: análisis genético multinivel del cáncer papilar de tiroides (CPT)}

Seudónimo: GOMAVI2019

Introducción: el cáncer papilar de tiroides (CPT) es un tumor diferenciado que se origina en las células foliculares. La incidencia, por razones hasta ahora poco claras, viene aumentando a nivel mundial, mientras que la mortalidad disminuye gracias al tratamiento quirúrgico y radioactivo; sin embargo, en Colombia la tendencia de mortalidad es positiva. Los factores de riesgo para desarrollar CPT son desconocidos, aunque la radiación ionizante se ha asociado de forma positiva. Las mutaciones en los genes RET y BRAF se han identificado como los principales eventos genéticos en $\mathrm{CPT}$, el primero mediante translocaciones y el segundo por la mutación puntual V600E. Se espera que otros genes estén involucrados en el proceso carcinogénico mediante diversos mecanismos mutagénicos, entre ellos las aberraciones cromosómicas.

Objetivos: en Colombia, la epidemiología molecular de estas alteraciones se desconoce; por tal razón, la presente investigación se propuso definir su frecuencia y descubrir nuevas alteraciones en una serie de pacientes colombianos con CPT por medio de las técnicas citogenética, reacción en cadena de la polimerasa con transcriptasa inversa (RT-PCR, por sus siglas 
en inglés) y secuencia masiva, esta última sobre 409 genes involucrados en el cáncer.

Resultados: a nivel citogenético, el $36 \%$ de los tumores mostraron alteraciones cromosómicas, como translocaciones recíprocas y desbalanceadas, trisomías o monosomías de carácter clonal. Ninguna de las muestras analizadas mostró rearreglos RET/PTC, mientras que el 87,5 \% fueron portadoras de alguna mutación puntual registrada en la base de datos
COSMID (Catalogue of Somatic Mutations in Cancer), por la técnica de secuencia masiva.

Conclusiones: este es el primer trabajo donde se aborda de manera integral y a diferentes niveles, con diversas técnicas, el descubrimiento de posibles alteraciones genéticas conductoras en el proceso carcinogénico en el CPT. Se concluye que el CPT es un modelo, interesante, importante y asequible para investigar la teoría mutacional del cáncer en múltiples pasos con perspectivas de aplicación terapéutica. 